\title{
Spectral analysis of tremor: understanding the results
}

\author{
Michael Gresty, David Buckwell
}

\begin{abstract}
Spectral analysis of a tremor record can sometimes produce a spectrum with multiple components of significant amplitude. The problem is to determine whether the presence of several peaks represents the coexistence of separate tremor mechanisms or be a consequence of fluctuations in the frequency or amplitude of a single tremor. The spectrum of a tremor whose frequency or amplitude vary and are independent has the recognisable pattern of a central carrier frequency with sidebands of equal amplitudes distributed symmetrically around the carrier. However, if tremor amplitude and frequency fluctuate and are not independent, (frequency proportional to amplitude or frequency inversely proportional to amplitude), the spectrum has a pattern of sidebands which are asymmetrical in amplitudes and may resemble the spectrum of the combined signal from different independent oscillators. The investigation of sidebands in spectra has been neglected in tremor studies and multiple irregular peaks on a tremor spectrum have sometimes been used wrongly as evidence for the coexistence of multiple tremor mechanisms or frequency components assumed to be concurrent.
\end{abstract}

Medical Research Council Human Movement and Balance Unit, Institute of Neurology, National Hospital, Queen Square, London M Gresty D Buckwell

This article is dedicated to Mr Edward Trinder, Chief Research Officer of the Medical Research Council Neuro-otology Unit who retired in the Autumn of 1989. His career was marked by great kindness to staff under his care and outstanding technical contributions to

understanding deafness and understan

Correspondence to: Dr Gresty, Medical Research Council Human Movement and Balance Unit, Institute of Neurology, National London WCIN 3BG, United Kingdom

Received 12 January 1990. Accepted 10 April 1990
As early as 1904 Gordon Holmes recognised that individual types of tremor, such as the rest tremor of Parkinson's disease or cerebellar action tremor, appear to be relatively fixed in frequency and speculated that the frequency of a tremor is specific to its pathophysiological origin. ${ }^{1}$ In contrast to frequency, the amplitude of tremor and the shape of the tremor are subject to wide fluctuations. In recent times, precise measurement of the characteristics of tremor, including frequency, has become widely available through spectral analysis which has been used to quantify tremor in clinical trials or as the bases of arguments concerning the nature of tremor mechanisms. Unfortunately, the results of spectral analysis can be as complicated in themselves as the raw tremor records. We draw attention to the problems of interpreting spectral analyses of tremor records and in particular to the circumstances under which multiple peaks may appear in tremor spectra. Nature of the tremor record: amplitude and
frequency modulation

Tremor is an approximately sinusoidal oscillation of a part of the body. The amplitude of the oscillation may fluctuate and this is termed amplitude modulation "AM". The frequency of the oscillation also varies. This is termed frequency jitter in physiology but here we will use the universal term frequency modulation "FM". The tremor waveform may be distorted, "harmonic distortion", which arises, in part, because of mechanical factors, and in part reflects the specific intensity and sequence in which various muscles are activated during the tremor cycle. These characteristics are illustrated in fig 1 showing the tremor in flexion/extension of the outstretched hand in a subject with essential tremor. The AM, FM and harmonic distortion of tremor may vary in an unlimited number of ways; thus for example, the amplitude may vary in a predictable way, waxing and waning at regular intervals ("spindling"); it may maintain an almost constant level or fluctuate randomly, or according to some combination of these.

For some tremors, frequency and amplitude are related as in the example of essential tremor given in fig 1 . The superimposed cycles of tremor show that as the time length of a cycle of the tremor decreases the amplitude also decreases; thus for this tremor, amplitude is inversely proportional to frequency within certain constraints. This characteristic of tremor has not been studied

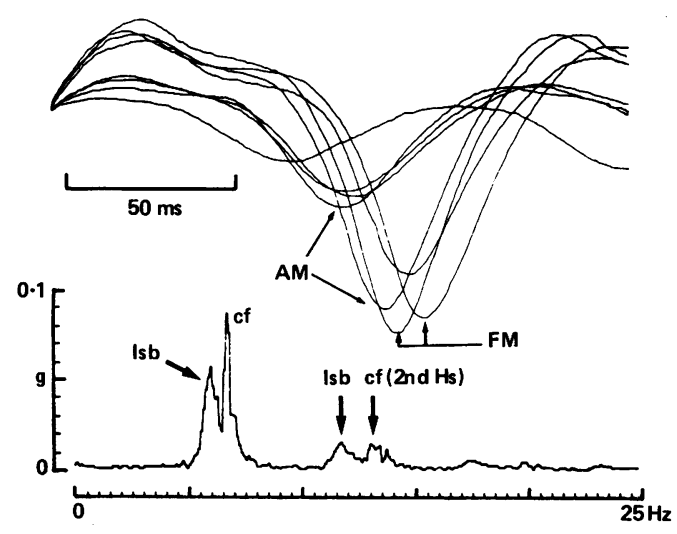

Figure 1 Accelerometric recordings and averaged spectrum of postural tremor of the hand in a 43 year old male with essential tremor. Individual cycles of tremor are shown superimposed by triggering from an

oscilloscope. As amplitude increases, the period of the tremor cycle elongates. The acceleration is scaled in units of "g" (earth gravitational field). lsb: lower sideband, cf: carrier frequency, $\mathrm{Hs}$ : harmonics. 

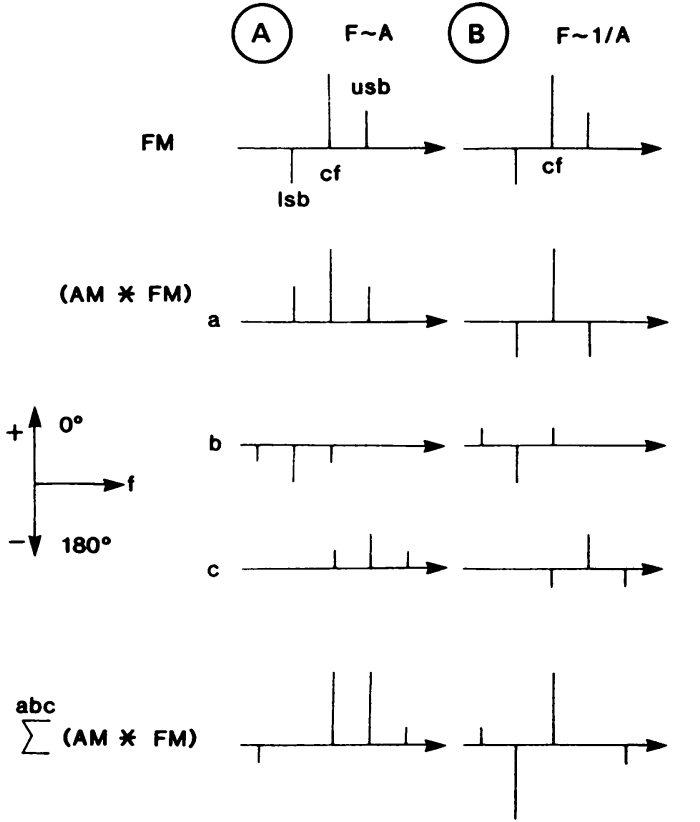

Figure $2 A, B$ Graphical representation of the frequency components of frequency and amplitude modulated tremor signals for frequency proportional to amplitude $F \sim A$ and frequency inversely proportional to amplitude $F \sim 1 / A$. Each of the bar pictures is $a$ spectrum. The horizontal axis in each case represents frequency. The vertical bars represent the individual terms of the Sine series which makes up a signal (see appendix). The frequency of each Sine component is shown by its location on the frequency axis. The coefficient of each term, that is, the size of each component, is shown by the length of the bars. The positive or negative sign of the coefficient is shown by the directions of the bars; $0^{\circ}$ for $+v e$ and $-180^{\circ}$ for $-v e$. The simplest case is shown of sinusoidal frequency and amplitude modulation. Frequency modulation of a sinusoidal signal produces the frequency components shown in the spectra labelled "FM" where "cf" is carrier frequency, "usb" is upper sideband and "lsb" is lower sideband. Amplitude modulation takes place on each of the components of the frequency modulated signal producing the frequency components shown in spectra labelled " $A M^{\star} F M a, b, c$ ". Thus: " $a$ " is the spectrum of amplitude modulation of the FM carrier frequency, " $b$ " is the spectrum of amplitude modulation of the FM lower sideband and " $c$ " is the spectrum of amplitude modulation of the FM upper sideband. Note that each of these resulting spectra have a central carrier frequency with an upper and a lower sideband. Since all these components coexist the resulting signal is their algebraic sum shown in the lowest traces labelled " $\Sigma a, b, c\left(A M^{\star} F M\right)$ ". Bars of equal amplitude but in opposite directions cancel when added together. Thus for FM $\sim A M$ (fig $2 A$ ) the lower sidebands of spectra $a$ and $b$ cancel each other. For $F M \sim 1 / A M$ (fig $2 B$ ) the upper sidebands of spectra a and $c$ cancel each other.

and yet is of crucial importance, for interaction between amplitude and frequency can give rise to an ambiguous spectrum.

Spectral analysis of combined FM and $A M$ waveforms

The spectra of AM and FM waveforms are explained in the appendix and shown graphically in fig 2 . The application to tremor is as follows:

Amplitude modulation and AM spectra: Tremor amplitude varies widely in a regular or irregular fashion. The waveform describing the amplitude variations is termed the "baseband signal". The spectrum of an amplitude modulated signal consists of a peak at the carrier frequency equal amplitude "sidebands" which are frequency components equally spaced above and below the carrier frequency.

If the amplitude of the tremor varies slowly over time (implying that the baseband signal is low frequency) the sidebands merge with the peak of the carrier frequency as shown in fig 3 . If the tremor spindles, for example fluctuates in amplitude every $2 s$, there are two distinct sidebands in the spectrum spaced $0.5 \mathrm{~Hz}$ above and below the carrier frequency. This is shown in graphs a, b, c of fig 2 which are AM spectra derived by amplitude modulation of the carrier frequency and of the upper sideband and the lower sideband of the FM signal.

Frequency modulation and FM spectra: Tremor varies in frequency about a typical centre frequency termed the "carrier frequency". The way in which the frequency varies can be described by a waveform, which can be regular or irregular, and is termed the "baseband signal". The spectrum of this signal consists of a peak at the carrier frequency of the tremor and equal amplitude "sidebands" which are frequency components equally spaced above and below the carrier frequency. The greater the variability in frequency of a signal, the more sidebands are produced. For example, the frequency of Parkinsonian postural or rest tremor varies within narrow limits of $+1-$ $0.5 \mathrm{~Hz}$ which produces only two important sidebands.

If the frequency of a tremor signal varies slowly over time (implying that the baseband signal is low frequency) the sidebands merge with the peak of the carrier frequency as shown in fig 3. If the tremor jitters, for example, fluctuates in frequency in two second epochs $(0.5 \mathrm{~Hz})$, then distinct sidebands appear on the spectrum spaced $0.5 \mathrm{~Hz}$ above and below the carrier frequency giving multiple peaks in spectrum. Important to the present argument is that the spectra of both the FM signal and the AM signal are the same except that the FM signal has a phase shift of $180^{\circ}$ in the lower sideband. This is shown by the downwards direction of the lower sideband of the FM spectra in fig 2 (see appendix).

Combined FM and $A M$ spectra: If the frequency modulation and amplitude modulation of a signal are unrelated then the spectrum typically has numerous upper and lower sidebands symmetrical in amplitude and evenly spaced in frequency about the carrier frequency. For the special cases where amplitude and frequency are related (an example is shown in the upper raw data traces of fig 1 where cycles of tremor with higher amplitudes have longer durations and therefore lower frequency) the sidebands become asymmetrical in amplitude as follows. The sideband and carrier frequencies produced by FM modulation become a complex carrier signal for the AM modulation, that is, the FM effectively generates three separate signals and amplitude 
Figure 3 The upper trace shows sinusoidal tremor with small, slow fuctuations in amplitude and frequency. The lower graph is the spectrum of this type of tremor. lsb: lower sidebands, $c f:$ centre frequency, usb: upper sidebands.
Figure 4 Tremor with harmonic distortion and moderately slow amplitude and frequency

fluctuations: (frequency proportional to amplitude). This type of tremor is typical of the rest tremor of Parkinson's disease. The asymmetry of the waveform produces harmonic distortion. The harmonic distortion. The
simulation assumes small amplitude fluctuations which occur typically at 2.5 s intervals with some statistical variability about this value from $1.5 \mathrm{~s}$ to 10s. H: harmonics.

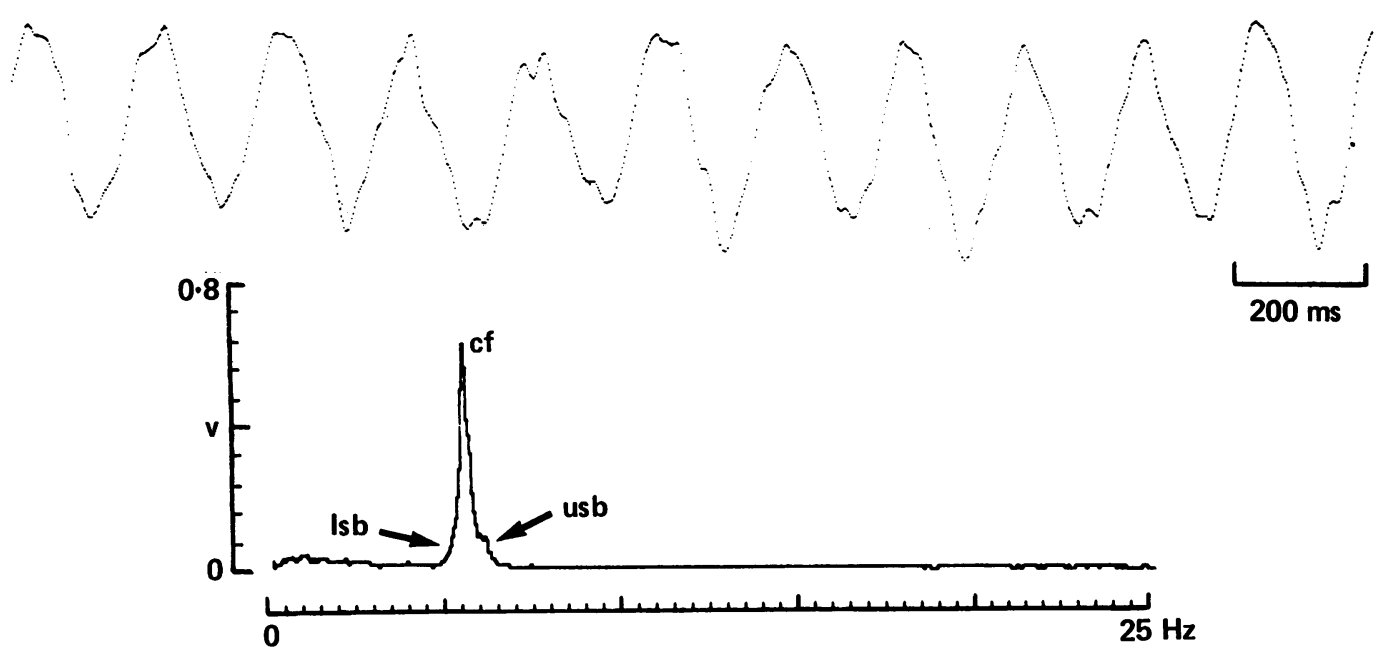

modulation of each of these takes place. If frequency is inversely proportional to amplitude, AM produces two upper sidebands at the same frequency but $180^{\circ}$ out of phase which cancel leaving only the lower sideband (see appendix). Conversely, if frequency is proportional to amplitude (for example, figs 3-5), AM produces two lower sidebands at the same frequency but $180^{\circ}$ out of phase which cancel leaving only the upper sideband. This process is shown in fig 2 for $F \sim A$ and $F \sim$ 1/A where the individual spectra $a, b$ and $c$ produced by amplitude modulation of each of the FM components are added together algebraically to produce the summed spectra shown in the lowermost traces, $\Sigma A M \star F M$ ( ${ }^{\star}$ indicates amplitude modulation of the frequency modulated signal).

Figures 3-5 illustrate the shapes of spectra which may be obtained from signals which fluctuate in frequency and amplitude and in which frequency and amplitude interact. The examples have been generated electronically, in simulation of tremor, so that we know a priori the nature of the signal. However, the properties of the signals are similar to those illustrated in fig 1 for real tremor. For the purposes of simulation signals have been generated so that frequency varies in proportion to amplitude. This relationship gives a dominant peak in the spectrum which is the carrier frequency together with an upper side band at a higher frequency (as shown in fig $2, \Sigma A M{ }^{\star} F M$ for $\mathrm{A} \sim \mathrm{F})$

\section{Are amplitude and frequency related in real} tremors?

The published records of tremor spectra suggest that amplitude and frequency can be related as shown by the following examples. Spectra of normal tremor associated with isometric tension in the finger whilst pressing a keyboard show marked asymmetrical upper sidebands ${ }^{2}$ suggesting a proportional relationship between amplitude and frequency of oscillatory instability. Some of the published spectra of the rest tremor of Parkinson's disease show sidebands present on the upper frequency side of the dominant tremor peak indicating that as the amplitude of the tremor increases there is a shift towards a higher frequency. ${ }^{34}$ This is also the case with certain examples of head tremor. ${ }^{5}$ Conversely spectra of the postural tremor of Parkinson's disease have shown asymmetrical sidebands present on the lower frequency side of the dominant peak ${ }^{4}$ indicating an inverse relationship between amplitude and frequency as in the example we have given of essential tremor. Spectra of isometric finger tension in patients with frequency components of which some may be sidebands and others, independent frequency components. In contrast, spectra of "red nucleus" tremor ${ }^{2}$ or ocular tremor ${ }^{6}$ (pendular nystagmus) show little FM or AM. It is not certain whether these suggestions are correct as the spectra are ambiguous (compare fig 5A, fig 6). cerebellar lesions ${ }^{2}$ show multiple significant

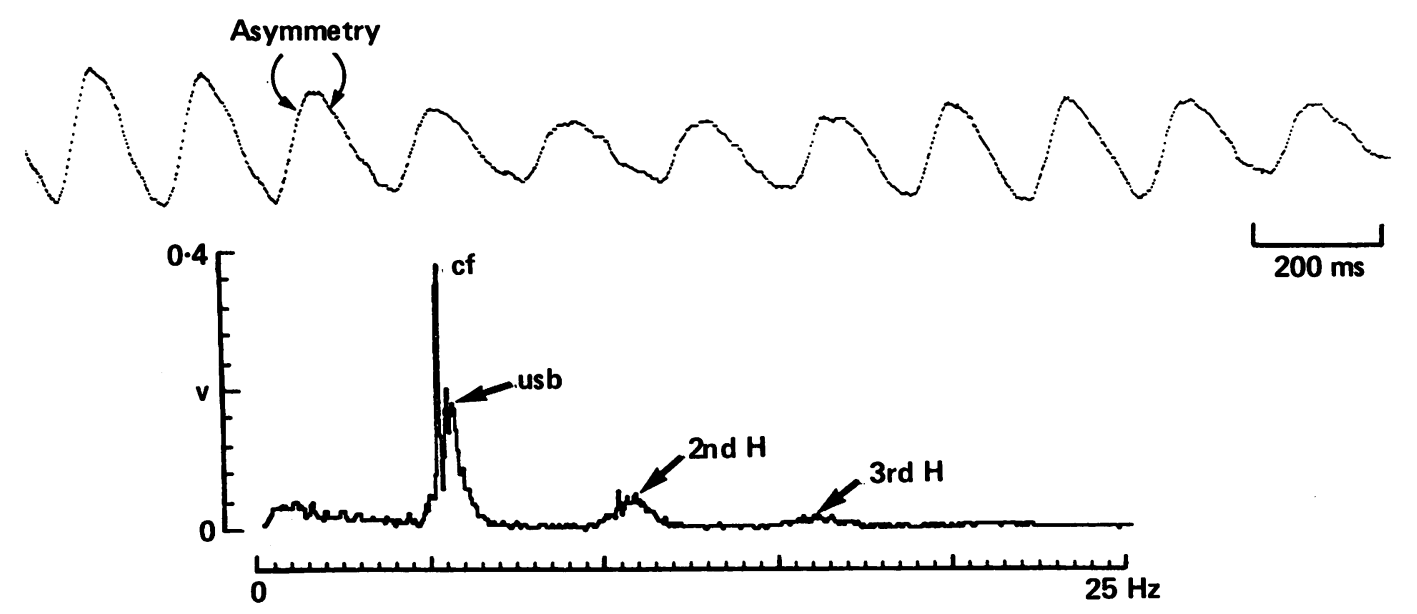


Figure 5 Examples of sinusoidal tremor with rapid fluctuations in amplitude and frequency (frequency $\sim$ amplitude). The appearance of this tremor is of spindling which arises because of wide variations in the value of the AM term in equation 1. A: sidebands (in this case on the lower side of the cf) almost disappear if the value of the AM term goes to zero; that is, the tremor stops momentarily. $B$, multiple sidebands appear if $F M$ causes wider variations in tremor frequency. The amplitude and number of sidebands of the spectra of this type of waveform depends upon the relative depth of the AM and FM. Note that amplitudes of the sidebands increase at the expense of the amplitude of the carrier frequency.

(A)
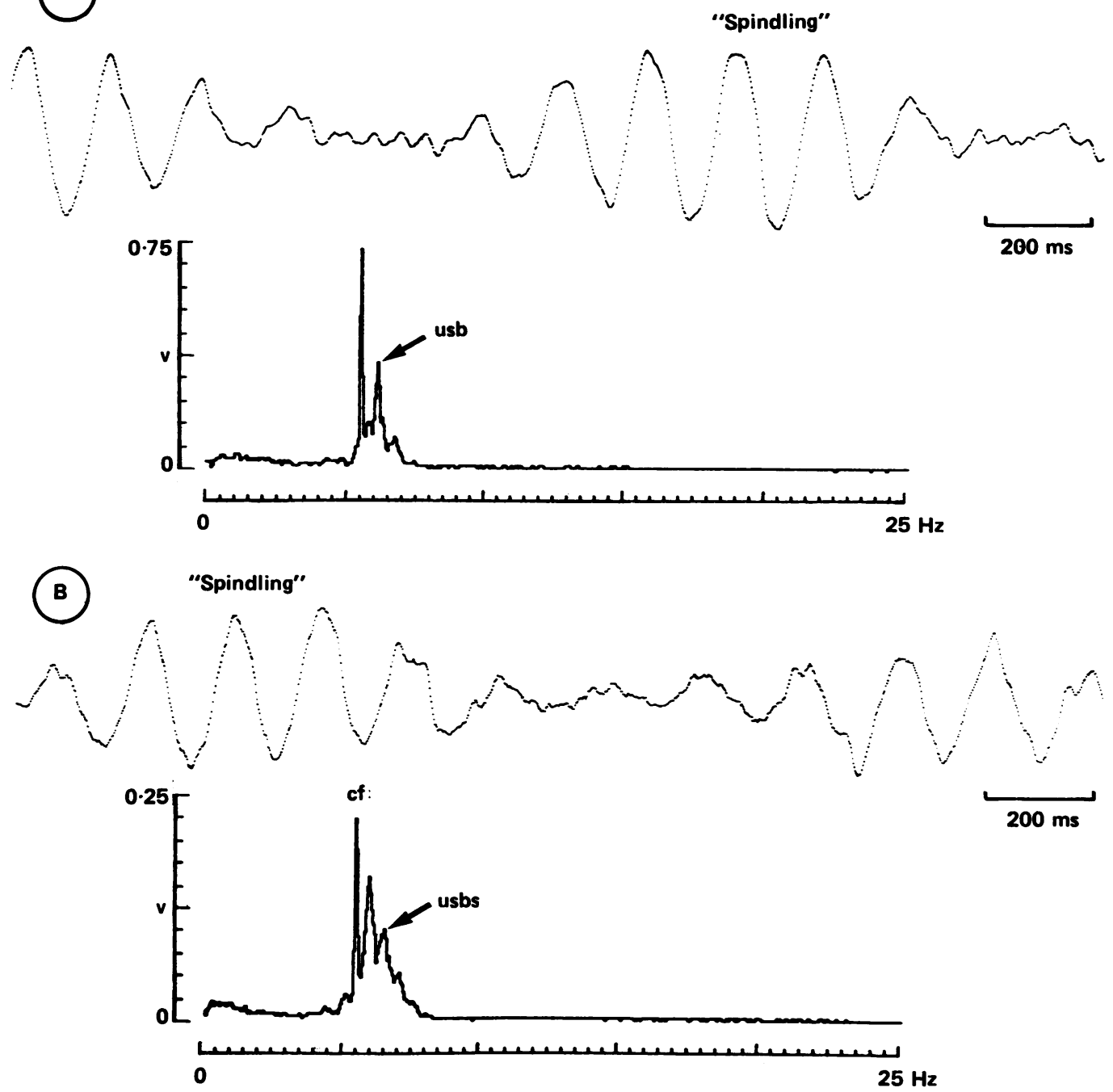

\section{Discussion}

The spectrum of tremor originating from a single oscillator can show multiple peaks of significant amplitudes. This has implications for tremor analysis and measurement.

The presence of two peaks in the tremor spectrum has been used as the basis for the argument that two separate tremor mechanisms are present. ${ }^{4}$ Since multiple peaks can be caused by simple variations in frequency and amplitude, the spectrum alone cannot suffice to support this argument and decisive evidence has to be sought elsewhere in the behavioural, pharmacological and physiological responsiveness of the various components of the tremor signal. The spectrum of two simultaneous tremors will have two peaks as in fig 6 and it may not be possible to decide whether the second peak is a sideband or not. Clues as to the presence of sidebands is that the multiple peaks, irrespective of amplitude, are all spaced in frequency equally above and below the
Figure 6 Tremor at two frequencies from independent generators. f1: 1 volt peak carrier with low level AM and $F M, f 2: 0.6$ volt peak carrier with low frequency, low level $A M$ and FM.

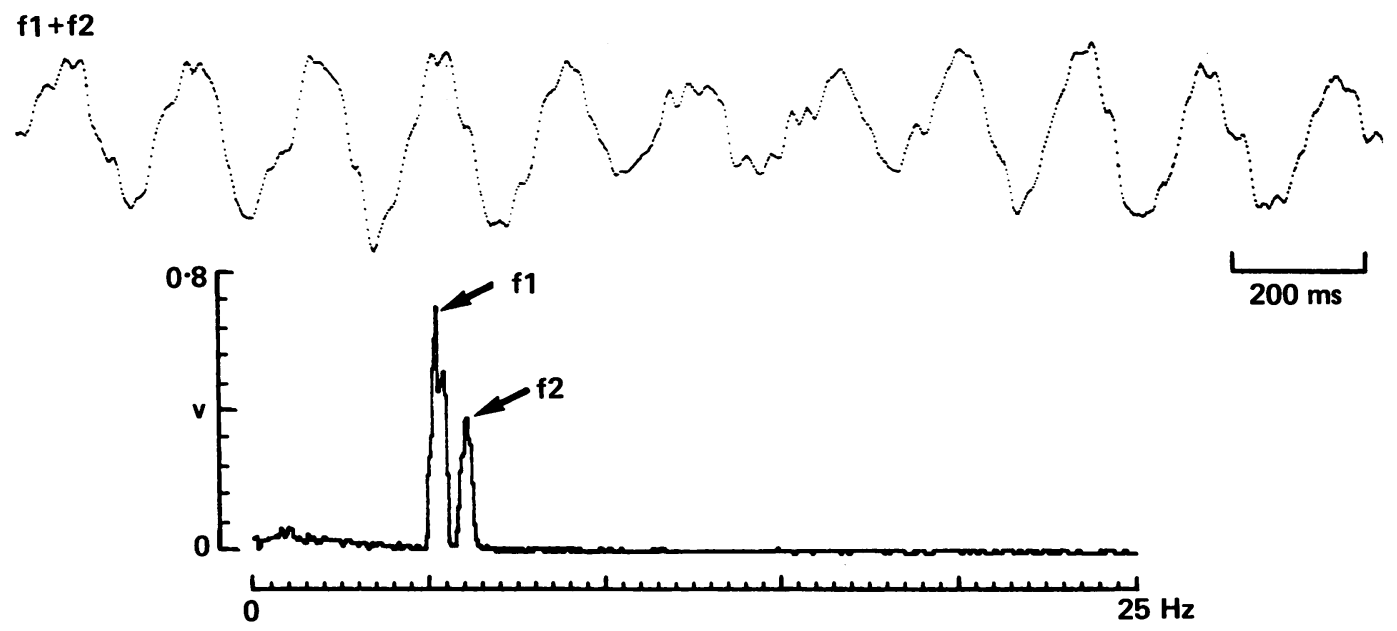


carrier frequency.

Little attention has been given to the multiple frequency components found in tremor spectra when measuring tremor amplitude, and authors have tended to take tremor amplitude as that of the dominant peak of the spectrum. A more suitable measurement of amplitude is the total power content in all significant frequencies of tremor present (see fig 5). A simple measurement, which would suffice for drug trials, would be the root-mean-square (rms) amplitude of the tremor signal over the time period of measurement if precautions are taken to exclude significant amounts of nontremulous movements. To build up a representative picture of tremor events it is unlikely that less than an average of 10 spectra would suffice. For the parameters of analysis used in the calculation of the spectra shown in this article 20 averages were required before a stable picture emerged. It is important that the rate at which the tremor signal is sampled must be high enough to resolve the highest frequency components of the motion and similarly that the number of samples acquired must be sufficient to detect the lowest frequency present in the modulating signal.

The nature of the tremor mechanism and the origins of amplitude and frequency variations and harmonic distortions are unknown. Thus attempts to start or stop a tremor in some way or entrain the tremor to some external perturbation (that is "resetting" or "driving" the tremor) might be influencing, to a greater or lesser extent, different parts of a rather complex and possibly widely distributed mechanism. A tremor will appear to be strongly influenced by a resetting stimulus only if the stimulus resets the primary oscillator and also establishes a constant period and amplitude for the ensuing tremor cycle. If the applied stimulus does not systematically influence the $A M$ and FM characteristics of the tremor then, on averaging, the tremor may not appear to be so strongly influenced by the perturbation. It may be, partly because of these considerations, that experiments on resetting tremor, for example in Parkinson's disease and essential tremor, have reported variable results. ${ }^{7}$

These comments are valid for the spectral analysis of all types of signals. Thus for example, spectral analysis of EMG activity of a tremor which shows distinct peaks has sometimes been assumed to indicate the presence of motor units firing at different frequencies. In fact the peaks may reflect fluctuations in the number of motor units recruited and jitter in the timing of their burst activity.

\section{TECHNICAL APPENDIX}

The equations used for simulation of tremor were those used in the mathematical treatment of modulated signals. ${ }^{8}$

Amplitude modulation: the equation for a sinusoidal signal, $f_{c}(t)$, whose amplitude is made proportional to the instantaneous amplitude of a modulating signal $f_{m}(t)$ is

$$
f_{c}(t)=\left[K+a \operatorname{Cos}\left(2 \pi f_{m} t\right)\right] \operatorname{Cos}\left(2 \pi f_{c} t\right)
$$

$\mathrm{K}$ is the unmodulated carrier signal amplitude.

For sinusoidal modulation the modulating signal $f_{m}(t)=a \operatorname{Cos}\left(2 \pi f_{m} t\right)$

thus:

$$
f_{c}(t)=K\left[1+m \operatorname{Cos}\left(2 \pi f_{m} t\right)\right] \operatorname{Cos}\left(2 \pi f_{c} t\right)
$$

where $m$ is the "depth of modulation" as defined as:

$$
\frac{\text { modulating signal amplitude }}{\text { unmodulated carrier amplitude }}=\frac{\mathbf{a}}{\mathbf{K}}
$$

equation 1 expands by trigonometric identities to

$$
f_{c}(t)=K\left[\operatorname{Cos}\left(2 \pi f_{c} t\right)+m \operatorname{Cos}\left\{2 \pi\left(f_{c}-f_{m}\right) t\right\} / 2\right.
$$$$
\left.+m \operatorname{Cos}\left\{2 \pi\left(f_{c}+f_{m}\right) t\right\} / 2\right] \ldots \text { equ 1a }
$$

This expression is the amplitude spectrum of the modulated carrier which consists of three frequency components: the carrier frequency $f_{c}$ and upper and lower side frequencies $\left(f_{c}+f_{m}\right)$ and $\left(f_{c}-f_{m}\right)$.

Frequency modulation: The equation for a sinusoidal signal, $f_{c}(t)$ whose carrier frequency is made proportional to the instantaneous amplitude of a modulating signal, $f_{m}(t)$, is $f_{c}(t)=A \operatorname{Cos}\left[2 \pi f_{c} t+b \operatorname{Sin}\left(2 \pi f_{m} t\right)\right] \ldots$ equ 2

Assuming that the modulating signal $f_{m}(t)$ is a single sinewave $\operatorname{Sin}\left(2 \pi f_{m} t\right)$ $\mathrm{A}$ is the amplitude of the modulated signal b is the "modulation index" defined as:

$$
\frac{\text { carrier frequency deviation }}{\text { frequency of modulating signal }}
$$

If the modulation index $b$ is less than 1 , as with tremor whose frequency deviation is typically not greater than $+/-0.5 \mathrm{~Hz}$, then the series expansion of equation 2 can be approximated to the equation

$f_{c}(t)=A\left[\operatorname{Cos}\left(2 \pi f_{c} t\right)-b \operatorname{Cos}\left\{2 \pi\left(f_{c}-f_{m}\right) t\right\} / 2\right.$
$\left.+b \operatorname{Cos}\left\{2 \pi\left(f_{c}+f_{m}\right) t\right\} / 2\right] \ldots$ equ $2 a$

This expression is the amplitude spectrum of the frequency modulated carrier which consists of three frequency components: the carrier frequency $f_{c}$ and upper and lower side frequencies $\left(f_{c}+f_{m}\right)$ and $-\left(f_{c}-f_{m}\right)$.

Comparison of equations la and $2 a$ show that the FM spectrum is equivalent to the $A M$ spectrum but with a phase shift of $180^{\circ}$, as shown by the negative sign of the lower side frequency.

These equations for frequency and amplitude modulation generalise to modulating signals with multiple frequency components (for example, bandwidth limited noise) in which case the amplitude spectrum of the modulated signal contains a band of frequencies, termed "sidebands", above and below the carrier.

Simulation of tremor: The equation used for simulation of tremor $f_{c}(t)$ for amplitude proportional to frequency combined equations 1 and 2 thus:

$f_{c}(t)=K\left[1+f_{m}(t)\right] \operatorname{Cos}\left[2 \pi f_{c} t+b f_{m}(t)\right]$

... equ 3

For brevity the formation of the sidebands in the spectrum of a signal of this form is shown in graphical form (fig 2). 
Implementation: For realistic simulation the function $\mathrm{f}_{\mathrm{m}}(\mathrm{t})$ was bandwidth limited noise. For amplitude inversely proportional to frequency the amplitude modulation term became [1 $\left.-f_{m}(t)\right]$. $K$ was set at an arbitrary amplitude of 1 volt; figures 3-5. The source of the baseband signal " $f_{m}(t)$ " was a Bruel and Kjaer 1024 signal generator. The signal was approximately bell shaped, bandwidth limited noise typically centred on a frequency equivalent to 0.4 or $0.7 \mathrm{~Hz}(-20 \mathrm{~dB}$ down at $+/-0.3 \mathrm{~Hz}$ for simulation of spindling or centre frequency of $0.2 \mathrm{~Hz},-20 \mathrm{~dB}$ down at $+/-1 \mathrm{~Hz}$ to simulate low frequency amplitude and frequency fluctuations.

The FM signal was generated by applying the scaled output of the Bruel and Kjaer generator to a KDP Electronic Systems 301A voltage controlled oscillator which generated the carrier. The carrier frequency $f_{c} t$ used in simulation was typical of tremor, for example, $5 \mathrm{~Hz}$ to simulate Parkinsonian rest tremor. The output of the oscillator was a centre frequency which was modulated in proportion to the addition of the input voltage from the generator. Signals were processed and scaled on a EAL 1000 analogue computer. Scaling was chosen such that amplitude was allowed to modulate from zero to full signal strength of one volt peak. Frequency was allowed to modulate so that the centre frequency of the first upper and lower sideband was within $0.5 \mathrm{~Hz}$ of the carrier frequency as is typical for human pathological tremor. Care was taken to avoid overmodulation and carrier phase reversal.

Spectra of the modulated signals were computed on a Solartron 1220 Signal Processor producing 500 spectral lines with a frequency resolution of $0.05 \mathrm{~Hz}$ and range DC to $25 \mathrm{~Hz}$. Spectra were obtained from averages of 20 separate time records of $20 \mathrm{~s}$ each. Each record was shaped by a Hanning window. The simulated tremor signals were synthesised in $100 \times$ real time. However, the parameters of analysis given are as they would be if the simulations were run in real time.

1 Holmes G. On certain tremors in organic cerebral lesions, Brain 1904;27:360-75.

2 Cole JD, Philip HI, Sedgwick EM. Stability and tremor in fingers associated with cerebellar hemisphere and cerebellar tract lesions in man. $J$ Neurol Neurosurg Psychiatry 1988;51:1558-68.

3 Dietrichson P, Engebretsen O, Fonstelien E, Hovland J. Quantitation of tremor in man. In: Desmedt JE, ed. Physiological tremor, pathological tremors and clonus. Proc Clin Neurophysiol, Vol 5. Karger Basel 1978; 90-94.

4 Findley LJ, Gresty MA, Halmagyi GM. Tremor the cogwheel phenomenon and clonus in Parkinson's disease. $J$ wheel phenomenon and clonus in Parkinson's
Neurol Neurosurg Psychiatry 1981;44:534-46.

5 Gresty M. Stability of the head. Movement Disorders 1987; 21:65-86.

6 Ell JJ, Gresty MA, Chambers BR, Findley LJ. Acquired pendular nystagmus: characteristics, pathophysiology and pharmacological modification. In: Roucoux A, Crommelinck $\mathrm{M}$, eds. Physiological and pathological aspects of eye movements. Dr DW Junk, The Hague $1982 ; 89-98$.

7 Lee RB, Stein RG. Resetting of tremor by mechanical perturbations: a comparison of essential tremor and Parkinsonian tremor. Ann Neurol 1981;10:523-31.

8 Dunlop J, Smith DG. Analogue Modulation Theory. In: Telecommunications Engineering. UK: Van Nostrand Reinhold, 1984:43-76. 